\title{
The spatial distribution of substellar objects in IC 348 and the Orion Trapezium cluster
}

\begin{abstract}
M. S. N. Kumar and S. Schmeja
Centro de Astrofísica da Universidade do Porto, Rua das Estrelas, 4150-762 Porto, Portugal

e-mail: [nanda;sschmeja]@astro.up.pt

Received 14 May 2007 / Accepted 22 June 2007

ABSTRACT

Context. Some theoretical scenarios suggest the formation of brown dwarfs as ejected stellar embryos in star-forming clusters. Such a formation mechanism can result in different spatial distributions of stars and substellar objects.

Aims. We aim to investigate the spatial structure of stellar and substellar objects in two well-sampled and nearby embedded clusters, namely IC 348 and the Orion Trapezium cluster (OTC), to test this hypothesis.

Methods. Deep near-infrared $K$-band data that are complete enough to sample the substellar population in IC 348 and OTC are obtained from the literature. The spatial distribution of the $K$-band point sources is analysed using the minimum spanning tree (MST) method. The $Q$ parameter and the spanning trees are evaluated for stellar and substellar objects as a function of cluster core radius $R_{\mathrm{c}}$. Results. The stellar population in both IC 348 and OTC display a clustered distribution, whereas the substellar population is distributed homogeneously in space within twice the cluster core radius. Although the substellar objects do not appear to be bound by the cluster potential well, they are still within the limits of the cluster and not significantly displaced from their birth sites.

Conclusions. The spatially homogeneous distribution of substellar objects is best explained by assuming higher initial velocities, distributed in a random manner and going through multiple interactions. The overall spatial coincidence of these objects with the cluster locations can be understood if these objects are nevertheless travelling slowly enough to feel the gravitational influence of the cluster. The observations support the scenario of substellar objects forming as "ejected stellar embryos". Higher ejection velocities are necessary, but net spatial displacements may not be needed to explain the observational data.
\end{abstract}

Key words. stars: low-mass, brown dwarfs - stars: formation - ISM: kinematics and dynamics - methods: statistical

\section{Introduction}

Observations of embedded clusters display the structure and mass segregation generally thought to reflect the structure of the molecular clouds from which they were born and the gravitational potential of the cluster (Lada \& Lada 2003). Massive stars are found to be located at the centres of the clusters and the radial distribution of cluster members roughly follow King models or power-law variations (Hillenbrand \& Hartmann 1998). These findings are generally based on observations sampling the stellar content of the clusters. With the advent of sensitive infrared detectors, the substellar content has been effectively unveiled, thereby allowing studies of brown dwarfs and planetary mass objects. In the rest of this paper we shall refer to brown dwarfs and free-floating planetary mass objects as BDs without distinction. The BDs are known to be particularly luminous in their early stages owing to which large groups of these objects are detected in near-infrared surveys of embedded clusters (Muench et al. 2003; Lucas et al. 2005).

The formation of substellar objects can be explained through at least five alternative mechanisms (Whitworth et al. 2007). One of the important mechanisms, the so-called "ejected embryos" scenario, advocates formation of BDs as a result of premature ejection of protostellar embryos from multiple systems. This "ejection scenario" attributes higher velocity dispersion and spatial spread to BDs in comparison to stellar objects (Reipurth \& Clarke 2001; Kroupa \& Bouvier 2003). Observations of the radial velocities of BDs and stars in Taurus and Chameleon star-forming regions indicate that there is no significant difference in the velocity dispersion between stars and BDs (Luhman et al. 2007). Young stars and BDs are also thought to be homogeneously mixed in the Trapezium cluster and Taurus molecular cloud. Many of these results are obtained by studying a few objects in localised regions of the cluster or in a region like Taurus where star formation occurs in relative isolation. In a deep nearinfrared study of the IC 348 cluster, Muench et al. (2003) analysed the radial distribution of point sources, which indicated a relatively homogeneous distribution of BDs in space compared to the stellar population that is bound to the cluster potential.

Structural analysis of embedded clusters has gained importance in recent years, since numerical simulations of the star formation process on the scales of molecular clouds have become available (e.g. Bate et al. 2003), and can be tested against observational results. Furthermore, to facilitate the structural analysis of observed data, quantitative methods of statistical analysis have also been developed. The nearest-neighbour method and the minimum spanning tree (MST) method (Cartwright \& Whitworth 2004) are two statistical methods that can be applied to observational data to analyse and quantify the physical parameters of clusters based on structural analysis. The structures of some star-forming clusters have been analysed using the MST method (Cartwright \& Whitworth 2004; Schmeja \& Klessen 2006).

IC 348 and the Orion Trapezium cluster (hereafter OTC) are two nearby, well-studied star-forming regions for which deep near-infrared data are available. The OTC in particular has been 
the target of several deep surveys that aimed to unveil the sample of BDs down to planetary-mass objects. In both IC 348 and OTC, the BD population is well-sampled. These are also richly populated clusters where a statistically significant sample of substellar objects have been catalogued. In this letter we analyse the structure of both IC 348 and OTC to study the variations in the stellar and BD populations.

\section{Data sets and analysis methods}

Near-infrared point source catalogs for the OTC were obtained from the Muench et al. (2002) and Lucas et al. (2005) studies via the on-line VizieR database. The Muench et al. (2002) data cover a region centred on the Trapezium cluster to a $10 \sigma$ detection limit of $K=18.1 \mathrm{mag}$ and include all the sources found by Hillenbrand \& Carpenter (2000). The Lucas et al. (2005) data obtained through FLAMINGOS on the GEMINI south telescope cover a region adjacent to the boundary of the Trapezium cluster, which suffers from relatively little contamination from the $K$-band nebulosity. We combined both the catalogs taking care of overlaps that resulted in a coverage of $\sim 0^{\circ} 1 \times 0^{\circ}$. In the overlapping regions between the two surveys we chose to use the magnitudes from Muench et al. (2002). Data for the IC 348 region were obtained from the FLAMINGOS survey described by Muench et al. (2003). The data cover an $\sim 21^{\prime} \times 21^{\prime}$ area centred on the IC 348 cluster to a depth of $K \sim 17$ mag.

Based on comparisons of pre-main sequence evolutionary tracks (Lucas et al. 2005) and luminosity function modelling (Muench et al. 2002), sources brighter than $K=14$ mag in the OTC can be considered stars and sources with $K>14$ can be considered BDs. Similarly, in IC 348, sources fainter than $K=$ 15 mag correspond to BDs complete to a mass limit of $0.04 M_{\odot}$, seen through an extinction of $A_{v} \sim 7$. These estimations take the effects of extinction and nebulosity into account, hence they represent a sample uncontaminated by such effects. We use this classification in the following analysis to distinguish stars and BDs.

Control field data were obtained from the 2MASS point source catalog. The control fields were selected by looking at large area $\left(5^{\circ}\right)$ IRAS $100 \mu \mathrm{m}$ maps and choosing a region of very low emission. Point sources within a circle of radius $15^{\prime}$ were extracted with the criteria of photometric quality flags better than "D" and without confusion (ccflag $=0$ ). The Orion control field is centred at $05^{\mathrm{h}} 24^{\mathrm{m}}-07^{\circ} 20^{\prime}$. In the case of IC 348 , the off-cloud control field is located at $03^{\mathrm{h}} 36^{\mathrm{m}}+35^{\circ} 00^{\prime}$; another field on the Perseus molecular cloud centred at $03^{\mathrm{h}} 39^{\mathrm{m}}+31^{\circ} 20^{\prime}$ was also examined. Although the 2MASS survey $100 \%$ completeness limit is at $K=14 \mathrm{mag}$, several reliable detections of $K=15-17 \mathrm{mag}$ are also found in the point source catalog at a lower $\sigma$ level.

We use the positions and magnitudes to analyse the spatial distribution of the objects by means of a minimum spanning tree, the unique set of straight lines ("edges") connecting a given set of points without closed loops, such that the sum of the edge lengths is a minimum (e.g. Gower \& Ross 1969). We derive the mean edge length $\ell_{\mathrm{MST}}$ as well as the parameter $Q=\bar{\ell}_{\mathrm{MST}} / \bar{s}$ by combining the normalised mean edge length and the normalised correlation length $\bar{s}$ as described in Cartwright \& Whitworth (2004) and Schmeja \& Klessen (2006). The $Q$ parameter quantifies the cluster structure and distinguishes between a centrally condensated cluster $(Q>0.8)$ and a cluster showing fractal substructure $(Q<0.8)$, while $\ell_{\text {MST }}$ can be seen as the mean distance between two neighbouring objects. We construct the MST using the algorithm of Prim (1957) implemented in an IDL routine.

\section{Results}

The MST method was applied on the photometric data to analyse the following:

a) The variation in the $Q$ parameter as a function of source magnitude (therefore source mass) selected to construct the tree. At every magnitude (intervals of $0.2 \mathrm{mag}$ ), $Q$ was evaluated by considering all sources brighter than the given magnitude. This variation was studied for the cluster field and the control fields.

b) The structure of the MST for stars and BDs separately. The resulting mean edge lengths $\ell_{\mathrm{MST}}$, along with their standard deviation and minimum and maximum values, were obtained for each tree. Projected separations less than $150 \mathrm{AU}$ (indicating source binarity) are neglected in computing $\ell_{\text {MST }}$ to remove their effect on the overall distribution. Beyond that, the edge lengths were examined inside and outside a radius that defined the cluster core boundaries.

In Figs. 1 and 2 we show these results for IC 348 and OTC. In both figures, panel a) shows the variation in $Q$ with magnitude, while panels b) and c) show the tree structure for stellar and substellar sources, respectively. They include the boundary limits of the cluster as defined by radial profile modelling. The $\ell_{\text {MST }}$ values for each zone enclosed by the boundaries, along with the statistics, are listed in Table 1.

It can be seen from Figs. 1a and 2 a that the $Q$ values peak at brighter magnitudes and show a turnover at $K=14$ mag for IC 348 and $K=11 \mathrm{mag}$ for OTC decreasing smoothly towards fainter magnitudes. In comparison, the data from the control fields do not show significant variations in the $Q$ paramater with magnitude. They yield values in the range of $Q \approx 0.72$, the value expected for a random distribution. The peak $Q$ values for IC 348 and OTC are 0.9 and 0.94 , respectively, indicating centrally condensed configurations. Following the findings of Cartwright \& Whitworth (2004) (condensation for $Q>0.8$, fractral substructure for $Q<0.8$ ), these results imply that sources fainter than $K=15.5 \mathrm{mag}$ (the point where $Q$ touches 0.8 in Fig. 1a) are distributed in space with a fractal structure while brighter sources are condensed more centrally in IC 348. The same intrepretation in OTC suggests a general central condensation that is only weak for the fainter sources.

The structures of the MST in Figs. 1b and 2b display clusters at the centre of the field while Figs. 1c and $2 \mathrm{c}$ display a homogeneous distribution of sources. The clustering can be seen as branches of smaller edge lengths at the centre spreading into branches of longer edge lengths away from the centre. It can also be seen that the clusters in Figs. $1 \mathrm{~b}$ and $2 \mathrm{~b}$ coincide well with the B star in IC 348 and with the Trapezium stars in the OTC cluster. The fainter sources do not show detectable variations in the tree structure across the field. These results are demonstrated more quantitatively in Table 1 . The linear lengths in Table 1 are derived from the angular lengths by assuming a distance of $315 \mathrm{pc}$ and 500 pc to IC 348 and OTC. The edge lengths for stars and BDs are listed individually for each cluster inside and outside the cluster full-width half maximum boundaries (also referred to as cluster core radius $R_{\mathrm{c}}$ ). In the IC 348 cluster, the boundaries of $5^{\prime}$ and $10^{\prime}$ represent the cluster core radius $R_{\mathrm{c}}$ and the limit at which the radial profile merges with the background. These values are adopted from Muench et al. (2002) where the radial distribution of sources is modelled. Similarly, a boundary of $R_{\mathrm{c}}=1.2$ is assumed for OTC based on the King model fits from Hillenbrand $\&$ Hartmann (1998). In IC 348, the $\ell_{\text {MST }}$ values for stars within $R_{\mathrm{c}}=5^{\prime}$ and outside vary by a factor of 1.9 , whereas they show 

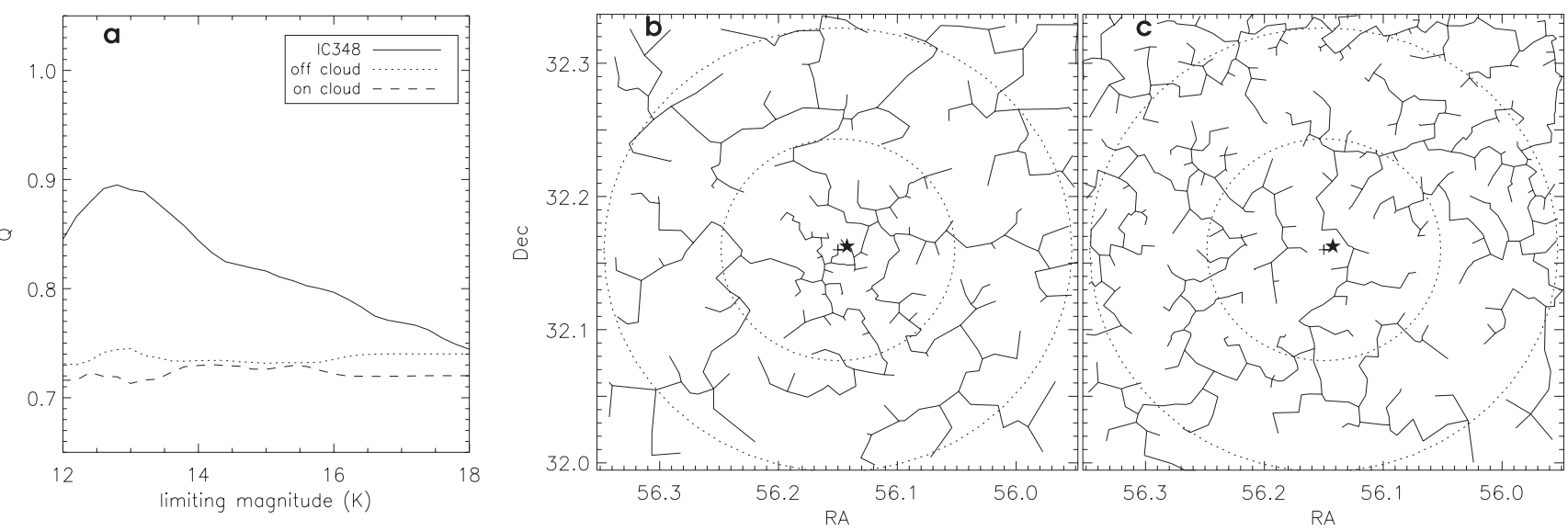

Fig. 1. IC 348: a) Plot showing the variation in the $Q$ parameter with magnitude of the point sources considered for the MST analysis. The solid line represents the curve for Flamingos $K$ band data complete to $K=18 \mathrm{mag}$. The dotted and dashed curves are for two control fields data obtained from 2MASS. b) The minimum spanning trees of stars (sources brighter than $K=15 \mathrm{mag}$ ). c) The minimal spanning tree for BDs (sources fainter than $K=15 \mathrm{mag}$ ). The inner and outer dotted circles have radii of $5^{\prime}$ and $10^{\prime}$ in $\mathbf{b}$ ) and $\mathbf{c}$ ). The B star in the centre is marked by a filled star symbol.
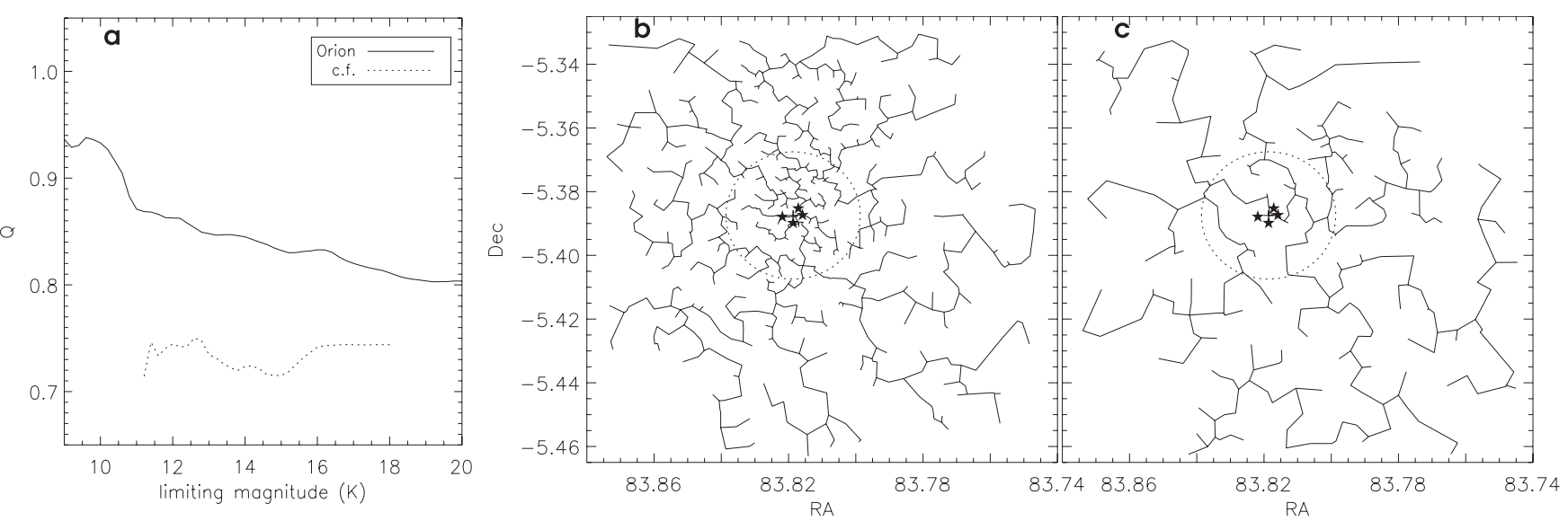

Fig. 2. Same as Fig. 1 for the Orion Trapezium cluster. In this case the sources brighter than $K=14$ mag are considered as stars. The dotted circle marks a radius of $1: 2$ representing the Trapezium cluster radius obtained by a King model fit. The star symbols indicate the positions of the Trapezium stars.

Table 1. MST parameters for IC 348 and OTC.

\begin{tabular}{|c|c|c|c|c|c|}
\hline Survey & $\begin{array}{r}\ell_{\mathrm{MST}} \\
10^{3} \mathrm{AU}\end{array}$ & $\begin{array}{r}\sigma_{\ell} \\
10^{3} \mathrm{AU}\end{array}$ & $\begin{array}{r}\ell_{\min } \\
10^{3} \mathrm{AU}\end{array}$ & $\begin{array}{r}\ell_{\max } \\
10^{3} \mathrm{AU}\end{array}$ & $\overline{n_{\star}}$ \\
\hline \multicolumn{6}{|l|}{ IC 348 } \\
\hline Stars $\left(<5^{\prime}\right)$ & 7.8 & 4.1 & 1.4 & 22.8 & 135 \\
\hline Stars $\left(>5^{\prime}\right)$ & 14.7 & 7.7 & 0.8 & 43.8 & 238 \\
\hline BDs $\left(<5^{\prime}\right)$ & 9.6 & 5.0 & 1.1 & 25.3 & 98 \\
\hline $\operatorname{BDs}\left(>5^{\prime}\right)$ & 9.5 & 5.1 & 1.0 & 36.3 & 536 \\
\hline \multicolumn{6}{|c|}{ Orion Trapezium } \\
\hline$\overline{\text { Stars }}\left(<1^{\prime} .2\right)$ & 2.5 & 1.3 & 0.4 & 6.5 & 228 \\
\hline Stars $(>1.2)$ & 5.4 & 3.4 & 0.16 & 26.1 & 570 \\
\hline BDs $\left(<1^{\prime} 2\right)$ & 5.8 & 3.4 & 0.5 & 13.7 & 49 \\
\hline BDs $(>1.2)$ & 8.4 & 5.0 & 0.16 & 26.9 & 258 \\
\hline
\end{tabular}

similar values for substellar objects (denoted as BDs). In the case of OTC, the $\ell_{\text {MST }}$ values of stars change by a factor of 2.12 and those of BDs by 1.4 within and outside the assumed cluster core radius of $R_{\mathrm{c}}=1$.2.

The above results are free of contamination due to binarity (as we excluded tree branches smaller than $150 \mathrm{AU}$ ) but include small $N$ clusters. A total of 19 sources were excluded in the OTC sample that imitated binary lengths. The comparisons inside and outside the cluster radius $R_{\mathrm{c}}$ show that the stars are centrally concentrated in both IC 348 and OTC clusters, whereas the BDs are distributed uniformly. In IC 348, the BDs are clearly homogeneously distributed within the radius of $10^{\prime}$, reproducing the results of Muench et al. (2003). The MST variation of BDs in OTC may indicate weak concentration at the centre, although the region examined here only encompasses the Trapezium cluster, which is the central part of the much larger Orion Nebula cluster (Hillenbrand 1997). Therefore, these variations show that, unlike stars, the BDs are not limited by the cluster potential in both IC 348 and OTC. The substellar objects are rather uniformly distributed in space, imitating a fractal configuration.

\section{Discussion}

The results of the analysis presented in the previous section has two significant parts:

a) The BDs are spread uniformly in space and not limited by the cluster potential well, whereas stars are bound by a welldescribed cluster potential. This can be explained if BDs in the cluster are seen as an $N$ particle system with higher initial velocities than the stars in random directions produced by multiple interactions at early times. The net result will be a relatively uniform distribution of space positions and 
velocities instead of being constrained by the gradient of the cluster potential well. Such a scenario of multiple objects and chaotic interactions is described well by numerical simulations such as those of Bate et al. (2003). However, this process is relevant only during the early evolution of the cluster (within $10^{5} \mathrm{yrs}$ ), thus setting up the "initial" conditions for BDs. Higher initial velocities are necessary for overcoming the cluster potential gradient and producing the homogeneous distribution of spatial locations. The natural effect of lowermass objects acquiring higher recoil velocities in comparison to higher-mass objects (Reipurth \& Clarke 2001; Kroupa \& Bouvier 2003) can result in BDs having the necessary ejection velocities. This is in accordance with the predictions of Kroupa \& Bouvier (2003), who computed higher dispersion in ejection velocities of $2-3 \mathrm{~km} \mathrm{~s}^{-1}$ for BDs.

b) The BDs appear slightly more centrally concentrated in the 1 Myr old OTC than in the 3 Myr old IC 348 cluster. While the BDs show a homogeneous distribution in space, they are still limited to the physical boundaries of the cluster or only slightly spread out. This is evident from the significant number of BDs in comparison to stars in both clusters (see last column of Table 1). It implies that the BDs have not traversed in space significantly away from the regions where they were born. The more uniform distribution in the 3 Myr old IC 348 cluster with respect to the 1 Myr old OTC imply that, with time, the BDs may indeed be moving away from the cluster centre, but the net displacement is not significant. Assuming higher initial velocities for BDs, the cluster potential that operates on the larger scale, will reduce their overall displacement away from the cluster centre. Goodwin et al. (2005) examine the spatial distribution of BDs produced by the decay of small $N$ stellar systems as expected from the embryo ejection scenario and show that a significant spatial spread of BDs with respect to stars may only occur after 5 Myr. Their predictions are consistent with the results of IC 348 and OTC.

The above results are therefore clear signatures of the "ejected stellar embryos" scenario (Reipurth \& Clarke 2001; Kroupa \& Bouvier 2003) as an important mechanism for the formation of brown dwarfs and free-floating planetary mass objects.

Several studies have been made to investigate the differences in spatial distribution of stars and BDs in star-forming regions. In the Chamaeleon I cloud, the distribution of BDs and lowmass stars was found to be similar (López Martí et al. 2004) and, in the Lupus cloud, BDs were found close to their birth sites (López Martí et al. 2005). In the Taurus molecular cloud, Briceño et al. (2002) examined the distributions of nearest neighbours to objects with spectral indices $\leq$ M6 and $>$ M6 and concluded that there were no significant differences in the spatial distribution of stars and BDs. Examining a smaller group of T Tauri stars, Martín et al. (2001) hinted at an anticorrelation between the density of stars and BDs in Taurus. Luhman (2006) examined an area of $225 \mathrm{deg}^{2}$ covering almost the entire Taurus molecular cloud and found no significant differences in the spatial distributions of BDs and stars.

The above studies were made because, according to the "ejected embryos" scenario, BDs may be expected to be significantly displaced from their birth locations. For example, assuming a 1D dispersion velocity of $1 \mathrm{~km} \mathrm{~s}^{-1}$, they should have moved $\sim 1$ pc away (corresponding to $7^{\prime} \approx 6 \times R_{\mathrm{c}}$ ) from the cluster in the 1 Myr old OTC or about 3 pc away (corresponding to $35^{\prime} \approx 7 \times R_{\mathrm{c}}$ ) in the IC 348 cluster (if they do not experience any retarding force from the cluster potential). However, as is evident from the data presented here, the BDs are indeed located within a region $\sim 2 \times R_{\mathrm{c}}$ in both clusters. Whether there is a significant concentration of BDs much farther away from the cluster cores in IC 348 and OTC is an issue that needs investigation and is beyond the scope of the data analysed here. However, following the example of Taurus, where Luhman (2006) examined the entire molecular cloud, significant populations of BDs away from the dense cores may not be expected.

The major difference between previous studies and this work is the advantage of large number statistics and space densities of point sources found in IC 348 and OTC. These number statistics have made it possible to effectively apply the MST technique to analysing the spatial distribution. In the absence of the MST analysis, IC 348 and OTC would imitate the results of Taurus, Cha I, or Lupus described above. Indeed in Fig. 2 of Luhman (2006), it can be seen that the BDs are sparsely populated, while stars are relatively clustered. MST analysis for Taurus may also show the kind of variations found here, but the IC 348 and OTC are clearly better data sets for effective application of the MST method, which is "statistical" in nature. In conclusion, we have shown that the near-infrared observations of the IC 348 and OTC embedded clusters are in accordance with the "ejected stellar embryos" scenario for the formation of substellar objects. Higher ejection velocities for substellar objects are essential, but their spatial displacements from the birth sites may not be necessary to explain the observed data.

Acknowledgements. We thank an anonymous referee for useful suggestions. MSNK and SS are supported by a research grant POCTI/CFE-AST/55691/2004 approved by FCT and POCTI, with funds from the European community programme FEDER. This research has made use of the SIMBAD and VizieR databases, operated at the CDS, Strasbourg, France. This publication makes use of data products from the Two Micron All Sky Survey, which is a joint project of the University of Massachusetts and the Infrared Processing and Analysis Center/California Institute of Technology, funded by the National Aeronautics and Space Administration and the National Science Foundation.

\section{References}

Bate, M. R., Bonnell, I. A., \& Bromm, V. 2003, MNRAS, 339, 577 Briceño, C., Luhman, K. L., Hartmann, L., Stauffer, J. R., \& Kirkpatrick, J. D. 2002, ApJ, 580, 317

Cartwright, A., \& Whitworth, A. P. 2004, MNRAS, 348, 589

Goodwin, S. P., Hubber, D. A., Moraux, E., \& Whitworth, A. P. 2005, Astron. Nachr., 326, 1040

Gower, J. C., \& Ross, G. J. S. 1969, Appl. Stat., 18, 54

Hillenbrand, L. A. 1997, AJ, 113, 1733

Hillenbrand, L. A., \& Hartmann, L. W. 1998, ApJ, 492, 540

Hillenbrand, L. A., \& Carpenter, J. M. 2000, ApJ, 540, 236

Kroupa, P., \& Bouvier, J. 2003, MNRAS, 346, 369

Lada, C. J., \& Lada, E. A. 2003, ARA\&A, 41, 57

López Martí, B., Eislöffel, J., Scholz, A., \& Mundt, R. 2004, A\&A, 416, 555

López Martí, B., Eislöffel, J., \& Mundt, R. 2005, A\&A, 440, 139

Lucas, P. W., Roche, P. F., \& Tamura, M. 2005, MNRAS, 361, 211

Luhman, K. L. 2006, ApJ, 645, 676

Luhman, K. L., Joergens, V., Lada, C. J., et al. 2007, in Protostars \& Planets V, ed. B. Reipurth, D. Jewitt, \& K. Keil (Tucson: Univ of Arizona Press), 443

Martín, E. L., Dougados, C., Magnier, E., et al. 2001, ApJ, 561, L195

Muench, A. A., Lada, E. A., Lada, C. J., \& Alves, J. 2002, ApJ, 573, 366

Muench, A. A., Lada, E. A., Lada, C. J., et al. 2003, AJ, 125, 2029

Prim, R. C. 1957, Bell Systems Tech. J., 36, 1389

Reipurth, B., \& Clarke, C. J. 2001, AJ, 122, 432

Schmeja, S., \& Klessen, R. S. 2006, A\&A, 449, 151

Whitworth, A., Bate, M. R., Nordlund, A., Reipurth, B., \& Zinnecker, H. 2007, in Protostars \& Planets V, ed. B. Reipurth, D. Jewitt, \& K. Keil (Tucson: Univ. of Arizona Press), 459 